\title{
An ontology model for a context-aware preventive assistance system: reducing exposition of individuals with Traumatic Brain Injury to dangerous situations during meal preparation
}

\author{
Marisnel Olivares* ${ }^{a, b}$, Sylvain Giroux*a,c, Pierre De Loor*d, André Thépaut*b, Hélène Pigot*a,c,e, \\ Stéphanie Pinard ${ }^{\dagger a, c}$, Carolina Bottari ${ }^{\dagger e, f}$, Guylaine Le Dorze $e^{\dagger e, g}$, Nathalie Bier ${ }^{\dagger} f^{\prime}$ \\ *a DOMUS, Département d'informatique, Université de Sherbrooke, Québec-Canada \\ ${ }^{*}$ bab-STICC / Télécom Bretagne, France \\ ${ }^{\dagger c}$ Centre de recherche sur le vieillissement, CSSS-IUGS, 036, Canada \\ ${ }^{*}$ Lab-STICC / ENIB, France \\ ${ }^{\dagger}$ Centre de recherche Interdisciplinaire en réadaptation du Montréal métropolitain (CRIR), Canada \\ ${ }^{t f}$ École de Réadaptation, Université de Montréal, Canada \\ ${ }^{\prime g}$ École d'orthophonie et d'audiologie, Université de Montreal, Canada \\ *\{marisnel.olivares, sylvain.giroux\}@usherbrooke.ca, ‘nathalie.bier@umontreal.ca
}

Keywords: Active and Assisted Living (AAL), contextawareness, ontology, Hierarchical Task Network (HTN), Traumatic Brain Injury (TBI).

\begin{abstract}
Severe traumatic brain injuries often decrease individuals' judgment and awareness. Ensuing cognitive impairments could then expose these individuals to dangerous situations. This paper presents a Preventive Assistance System (PAS) for meal preparation. PAS was designed to prevent a detected potential risk situation from progressing towards a situation that is dangerous for the user's safety. Its final aim is to avoid a drastic safety intervention by the autonomous Safety System. PAS provides preventive assistance to four out of the six potential risk situations identified by an interdisciplinary team. It relies on a model of assistance, communication, and smart homes. This model is described in a taxonomy implemented in OWL 2. The preventive assistance model is inspired from Hierarchical Task Network (HTN) and the communication model is inspired by the speech act theory. While observing the user, the generation of a preventive assistance tree is guided by the Instrumental Activities of Daily Living (IADL) being executed and the detection of potential risk situations. The preventive prompts - jingles, pictograms, voice messages, written messages...- are provided gradually, proceeding from implicit/generic to explicit/specific, until the user successfully reacts to avert the danger.
\end{abstract}

\section{Introduction}

Each year in Canada, around 100000 people are victims of a Traumatic Brain Injury (TBI) [1]. TBI severity is generally rated as mild, moderate, or severe [2]. The severity influences the physical, cognitive, and emotional deficits exhibited by the person [3]. A severe TBI can cause serious cognitive impairments, such that persons would require constant assistance to perform Instrumental Activities of Daily Living (IADL) — preparing meals, shopping for groceries...-, impacting their capacity to live independently [4]. Moreover cognitive sequelae can affect their judgment and awareness, impairing their decision-making abilities [5]. Therefore, while performing IADLs, they can be exposed, or expose themselves, to dangerous situations such as fires, electrocution, burns, cuts, intoxications, and so on.

Assistive Technologies (AT) seek to improve the functioning in everyday activities of people with cognitive deficits [6]. Ideally ATs should foster autonomy while ensuring safety. Meal preparation is a key activity for keeping people at home. It favours self-esteem and helps in maintaining social participation too [7]. However meal preparation is a complex activity relying on high-level cognitive functions [8] that also entails many hazards. Hence, we designed and implemented ATs that help individuals with severe TBI resume independent and safe meal preparation in a smart home [9]. These ATs are made of three independent though intertwined systems. On the one hand, COOK guides users $^{1}$ during meal preparation. COOK also provides information and hints on safe behaviours in a kitchen. On the other hand, an autonomous Safety System (SS) ensures users' safety, primarily by switching off the stove and informing caregivers on the occurrence of precise hazardous situations. In between stands a Preventive Assistance System (PAS) whose role is to detect a potentially dangerous situation, warn the users, and assist them to avoid its evolution into a dangerous one where the SS would intervene.

This paper will focus on PAS. First, we explore related works with respect to meal preparation, safety and prevention (§2). Observing that no system is addressing context-aware personalized assistance for prevention where it is the user that has to take the remedying actions, we developed such a system, named PAS, its architecture, and its main components (§3). Then, we present the methodology (§4) followed to identify the relevant potential risk situations $(\S 5)$ and to build a formal model describing assistance, communication, and

\footnotetext{
${ }^{1}$ Henceforth "users" will refer to individual(s) with severe TBI which are the primary users of the ATs. Caregivers are secondary users that may be involved in some operations.
} 
smart homes (§6). We then illustrate how this model can lead to effective preventive assistance thanks to personas. Preventive assistance model is instantiated for each one after the detection of the same potential risk situation (\$7). Finally, a brief conclusion discusses the contribution of our work and outlines future work $(\S 8)$.

\section{Related work on ATs for meal preparation, safety, and prevention}

Many research efforts in AT development targeted meal preparation. For one, the ambient kitchen project studied the prompting of people in the early stage of dementia in multistep tasks, more precisely food and drink preparation [10]. A real kitchen was replicated in a lab. The ambient kitchen prototype can be used as a design tool, an observation tool to collect data for activity recognition, and a test and evaluation tool. The ambient kitchen environment integrated data projectors, cameras, RFID tags and readers, objects mounted on accelerometers, and under-floor pressure detectors. Though very interesting, the project did not address prevention and risks. Also, some of the sensors used in the ambient kitchen may be considered as privacy-invasive [11] and many of them cannot be easily installed in a real home.

Coronato et al. (2013) used situation-awareness to detect the anomalous situations in a kitchen environment. First order logic was used to define the entities of interest in the kitchen. An alarm informs users when an anomaly is detected. Nonetheless no details are provided on the types of alarms or the content used to explain the detected anomaly [12].

Mahajan (2013) used smart sensing and prompting technologies to guide individuals with cognitive impairments in everyday kitchen tasks [13]. A network of sensors feeds activity recognition. Prompting strategies guide the user to the completion of a task. Safety is ensured during and after the use of kitchen appliances: appliances can be automatically switched off in case a safety problem arises. However prevention is not addressed: the user cannot take corrective actions by himself or herself.

These researches pinpoint still open issues and the lack of a formal model for preventive assistance in meal preparation:

- How to describe and detect anomalous and dangerous situations?

- How to manage safety issues? When and how to prompt?

- Which sensors are necessary, sufficient and acceptable?

- What should be the relation between meal preparation (multi-step kitchen tasks), assistance, safety, and prevention systems?

\section{PAS: a Preventive Assistance System}

Existing ATs for meal preparation have limits that preclude their deployment in real homes. One limit is the absence of preventive assistance to reduce user exposure to dangerous situations. Another is their limited capacity to personalize the assistance to the individual's specific needs. Individuals with severe TBI exhibit a wide variety and range of cognitive difficulties. Each user is unique and the cognitive support each person needs and the interaction mode each person prefers will vary from one to another. Therefore, we need a preventive assistance model that captures each user's idiosyncrasies, permits the personalization of the preventive assistance and provides context-aware assistance with respect to hazards related to meal preparation. To address these problems, we designed PAS. PAS provides personalized preventive assistance promptings [14] to the users in order to reduce their exposure to dangerous situations during meal preparation. Artificial Intelligence techniques allow adaptation of the system to the user's occupational profile [15], through a formal specification of the system elements, goals, and inference rules [16]. Hence, an occupational therapist configures the user's occupational profile and sets the user's preventive assistance model, e.g. using an external strategy, user's prompting preferences (visual or/and audio), and the prompts contents.

While the user is cooking, when necessary, PAS has to progressively provide prompts to the user about the presence of a potential risk situation that needs to be solved over a predefined time limit. To do so, context-awareness [17] helps to deliver relevant cues at the right time. PAS is composed of three subsystems: Supervision, Communication, and Assistance. Supervision deals with the recognition of potentially hazardous situations. A non-invasive sensor network installed in the user's home captures data about the user's real-time actions. The user is not required to wear any sensors. Communication manages actuators (speaker(s), screen(s), TV light(s), etc.), selecting and activating relevant ones according to the user's location and preferences to transmit prompts. Assistance, a personalized preventive assistance tree, is gradually instantiated as a response to a detected potential risk situation, the user's cognitive capacities and preferences, as previously configured in the user's occupational profile.

PAS, COOK and SS all rely on the sensor and actuator infrastructure, but the role of each is both distinct and clearly defined. The cooking assistant COOK is a cognitive orthotic designed to use ambient intelligence to provide cognitive assistance to individuals with severe TBI to perform meal preparation at home. COOK can present to the user a declarative version of the safety rules to respect. The autonomous SS is activated when the COOK assistant is launched. It monitors the user's actions in the environment. If this system detects a preconfigured situation that is dangerous for the safety of the user, then it directly reacts by disabling the electrical appliance(s) related to the detected situation. SS thus applies the rules literally. The role of PAS is to help the user to resolve the situation before SS switches everything off.

\section{An interdisciplinary process}

PAS could not have been developed without a true collaboration between experts of various disciplines ${ }^{1}$,

\footnotetext{
${ }^{1}$ In the authors list, $\mathrm{a}^{\dagger}$ identifies researchers in health and clinical sciences (occupational therapy, speech and language pathology, neuropsychology, and physiotherapy) and ${ }^{*}$ identifies researchers
} 
caregivers, and users. First, this interdisciplinary team identified potential risk situations that could compromise users' safety during meal preparation. A literature review started the process. Then a workshop involving the Fire Department of Sherbrooke (Canada) was organized. Finally, open discussions lead to the definition of safety rules $(\$ 5)$.

Second, a formal representation of the preventive assistance model was defined (§6). This model relies on preventive assistance taxonomy, and its semantics, that delineate the application domain. This taxonomy and its semantics are then used to generate a preventive assistance tree, based on the detected potential risk situation, and the user's occupational profile.

\section{Identification of the potential risk situations}

In PAS, potential risk situations to be monitored during meal preparation are described as a context that includes the associated parameter(s) with trigger value(s) that must be monitored, the required sensor type involved in the detection of the situation, and the time limit after which the situation is expected to become dangerous (Table 1). The Fireman and five Occupational Therapists, members of the project, validated them.

Then a priority was assigned to each situation. The priority assignment was based on three factors: 1) dangerousness; representing a real danger to the user's safety, 2) implementation technical constraints; representing our capacity to adequately recognize the situation with the available sensors installed in the user's home, and 3) personalization; representing the user's cognitive assistance needs. Therefore, in this paper, we focused on the potential risk situations labeled as priority 1 . These situations are related to the use of the stove.

\begin{tabular}{|c|c|c|c|c|}
\hline 氞 & 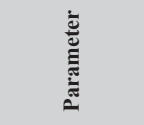 & 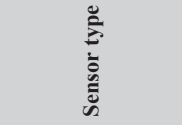 & 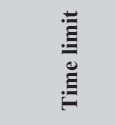 & $\stackrel{3}{=}$ \\
\hline $\begin{array}{l}\text { 1. Exiting home while the } \\
\text { oven is active }\end{array}$ & $\begin{array}{l}\text { Oven temp } \\
\leq 400^{\circ} \mathrm{F} \\
>400^{\circ} \mathrm{F}\end{array}$ & $\begin{array}{l}\text { Electromagnetic }+ \\
\text { temperature }+ \\
\text { motion }\end{array}$ & $\begin{array}{r}15 \text { minutes } \\
0 \text { minutes }\end{array}$ & 1 \\
\hline $\begin{array}{l}\text { 2. Exiting home while a } \\
\text { hotplate is active }\end{array}$ & $\begin{array}{l}\text { Hotplate } \\
\text { active }\end{array}$ & $\begin{array}{l}\text { Electromagnetic }+ \\
\text { temperature }+ \\
\text { motion }\end{array}$ & 2 minutes & 1 \\
\hline $\begin{array}{l}\text { 3. Leaving the oven } \\
\text { without supervision }\end{array}$ & $\begin{array}{l}\text { Oven temp } \\
<300^{\circ} \mathrm{F} \\
\leq 400^{\circ} \mathrm{F} \\
>400^{\circ} \mathrm{F}\end{array}$ & $\begin{array}{l}\text { Electromagnetic }{ }^{+} \\
\text {temperature }+ \\
\text { motion }\end{array}$ & $\begin{array}{l}60 \text { minutes } \\
30 \text { minutes } \\
10 \text { minutes }\end{array}$ & 1 \\
\hline $\begin{array}{l}\text { 4. Leaving stove hotplate } \\
\text { without supervision }\end{array}$ & $\begin{array}{l}\text { Hotplate } \\
\text { temp } \\
\leq \text { medium } \\
>\text { medium }\end{array}$ & $\begin{array}{l}\text { Electromagnetic }+ \\
\text { temperature }+ \\
\text { motion }\end{array}$ & $\begin{array}{r}10 \text { minutes } \\
2 \text { minutes }\end{array}$ & 1 \\
\hline $\begin{array}{l}\text { 5. Leaving an active } \\
\text { hotplate(s) empty for } \\
\text { several minutes }\end{array}$ & $\begin{array}{l}\text { Hotplate } \\
\text { temp } \\
>0{ }^{\circ} \mathrm{F}\end{array}$ & $\begin{array}{l}\text { Load cell }+ \\
\text { temperature }+ \\
\text { time }\end{array}$ & 2 minutes & 2 \\
\hline $\begin{array}{l}\text { 6. Forgetting to wash } \\
\text { hands before cooking }\end{array}$ & $\begin{array}{l}\text { kitchen tap } \\
\text { open }\end{array}$ & Flowmeter & 5 minutes & 3 \\
\hline
\end{tabular}

These situations correspond to the rules that the Preventive Assistance System must monitor. When one of

in computer sciences. Researchers in ergonomics and designers were also involved. these situations is detected, the assistance model is instantiated as an assistance tree providing cognitive support for the user to help prevent that the situation reaches the time limit.

\section{Formal representation of the preventive assistance model}

Once risk situations were identified, we defined a formal representation of the preventive assistance. Two processes guided its design: 1) the definition of a taxonomy model and its semantics to delineate our research domain, 2) the definition of the main principles required to generate a preventive assistance tree.

\subsection{Preventive assistance taxonomy}

The preventive assistance taxonomy was implemented in the OWL 2 Web Ontology Language [18] using the Protégé editor [19]. It contains the classes and their inter-relationships and properties given the semantics of the application domain. The model contains 5 main classes: Home, HomeDevice, Sensor, Actuator, PreventiveAssistance which are related to other classes such as: User, AssistanceLevel, AssistanceStep, Message, MessageType, Situation, etc.

\subsubsection{Modelling smart homes}

First, we need to model as logical entities the different areas of the Home where the User will execute the $I A D L$ (meal preparation) (FIGURE 1). Space is divided into HomeAreas. This allows us to determine in which HomeArea the HomeDevice(s) (FIGURE 2), Sensor(s) (FIGURE 3), and Actuator(s) (FIGURE 4) are located, as well as to detect the User location changes during the IADL execution.

The preventive assistance model will for instance use this information to decide when a user is not monitoring pans on the stove. The HomeArea granularity allowed us to easily define and identify the sensors required to detect a potential risk situation for the user's safety. Then, it will be easier to dynamically generate a personalized preventive assistance tree (FIGURE 5) which prompts the user with the corrective action(s) to execute in order to solve a detected situation. Inferences can then be made to determine where, and which actuators to choose, to transmit the prompts for the user through multimodal interfaces [20] based on the user's current location.

\subsubsection{Modelling assistance}

The preventive assistance model has to describe and reason on who is the user, what are the risk situations to monitor, and how to assist when necessary. Thus the PreventiveAssistance class is related to the following classes:

- The User class represents the individual with severe TBI whom we want to assist. Actually a User has just one AssistanceLevel (General, Intermediate or StepbyStep) corresponding to his/her level of impairments;

- The PotentialRiskSituation class establishes the sensor values to monitor and the time interval inside which 
preventive assistance can be provided to the User. When the sensor trigger values are reached, the situation becomes active. It determines the message content used to prompt the User;

- A message can be uttered in many different ways. If not understood by the user, it can be reformulated with more insistence. The AssistanceStep class represents this process, specifying, in particular, the number of interventions and variations over a determined period of time. Due to the cognitive impairments of the target population, we restricted AssistanceStep $(s)$ to a maximum of 3 messages at a time.

\subsubsection{Modelling communication}

Finally, assistance messages and prompting have to be modelled to find concrete means of expressing them within the smart home. First, the Message class represents the means chosen to transmit information to the User. Five prompting types are proposed in our model: Jingle, Voice, Bright, Pictogram, and Text. A recorded voice message option is proposed as the user's attention can be enhanced when hearing a familiar voice (own voice or a significant other's voice) [21]. The MessageType class represents the means to provide a progressive preventive assistance to the user. It was inspired from the speech act theory [22]. Two main categories of message types were specified: ImplicitMessageType (Jingle, Bright, and Pictogram) and direct or indirect ExplicitMessageType (Voice or Text).

Second, a prompting mechanism was designed to progressively increase the level of guidance offered by the preventive assistance from implicit to explicit or from indirect to direct promptings over the time limit of a detected potential risk situation. For instance, "Time to monitor your cooking?" is an indirect explicit message, while "Go monitor your cooking!" is a direct explicit one. The prompting mechanism model proposes default prompting hierarchies that can be chosen according to the user's AssistanceLevel and the MessageType. Thus, a General or Intermediate AssistanceLevel can choose to provide just one isolated ImplicitMessageType or a sequential combination of messages starting with IndirectExplicit and ending with DirectExplicit. On the other hand, the StepByStep AssistanceLevel always uses ImplicitMessageType in combination with DirectExplicit ones. The use of Jingle prompting is proposed by default for each of the three AssistanceSteps. Jingles can express three alert levels: remind, risk, and danger. Each alert level is associated with a volume intensity (low, medium, and high). Indeed, the prompting mechanism is flexible and can be adapted and personalized to the user's prompting preferences stated in his/ her occupational profile, e.g. only use Text or only use Pictograms for prompting.

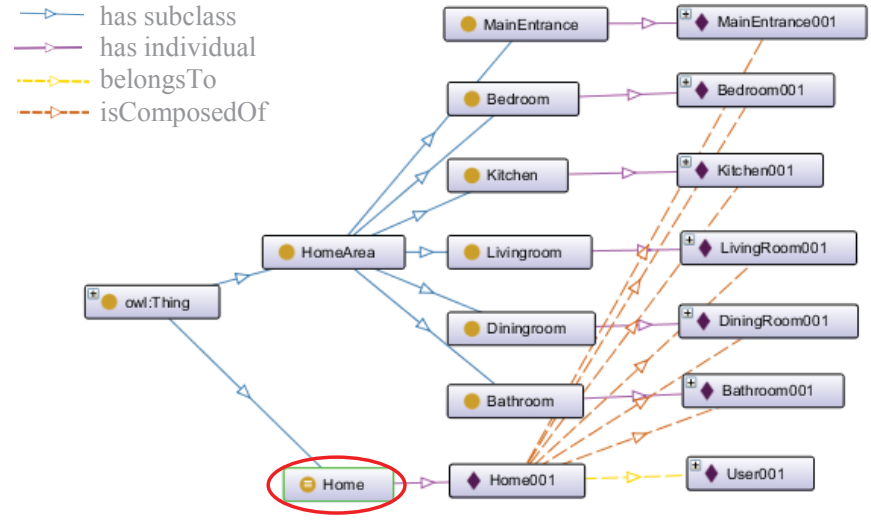

FIGURE 1: Definition of the Home and HomeArea classes in Protégé

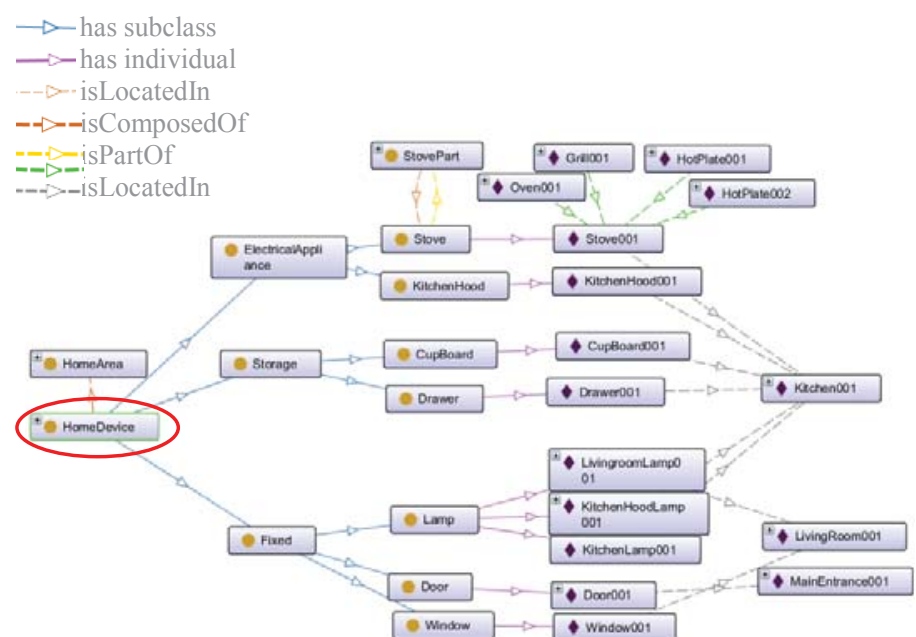

FIGURE 2: Definition of HomeDevice class in Protégé

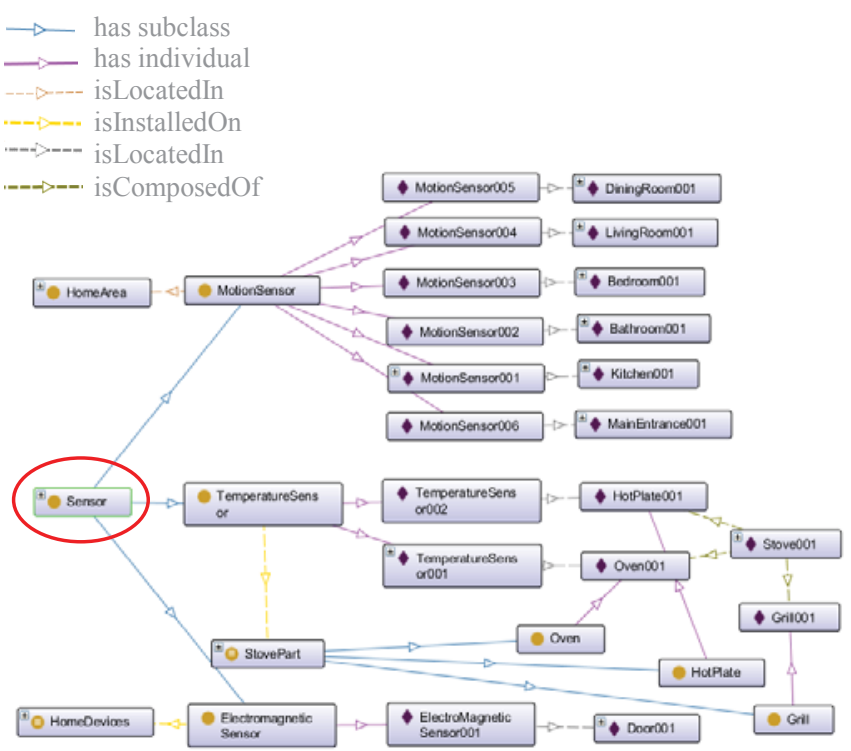

FIGURE 3: Definition of the Sensor class in Protégé 


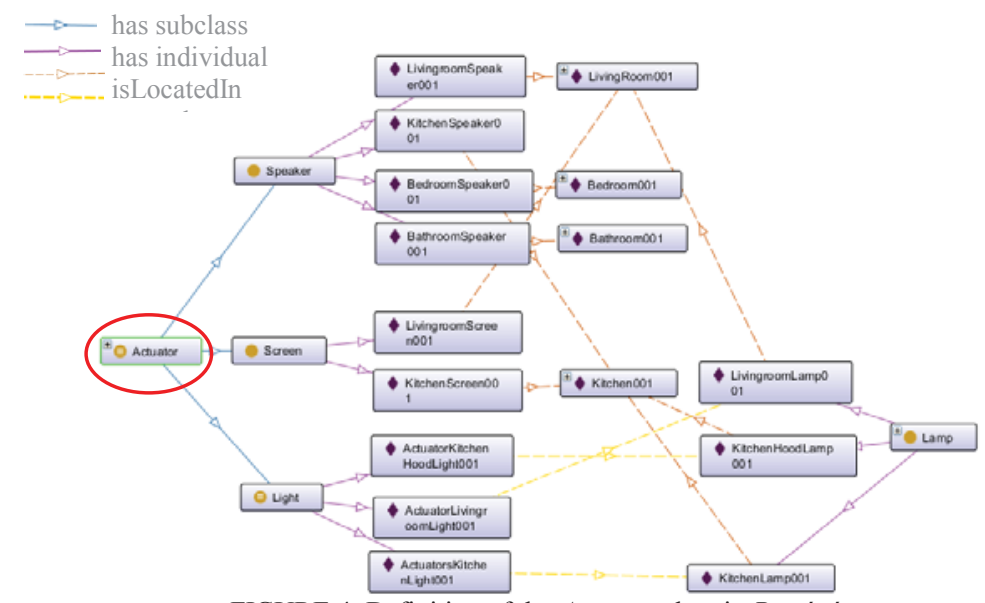

FIGURE 4: Definition of the Actuator class in Protégé

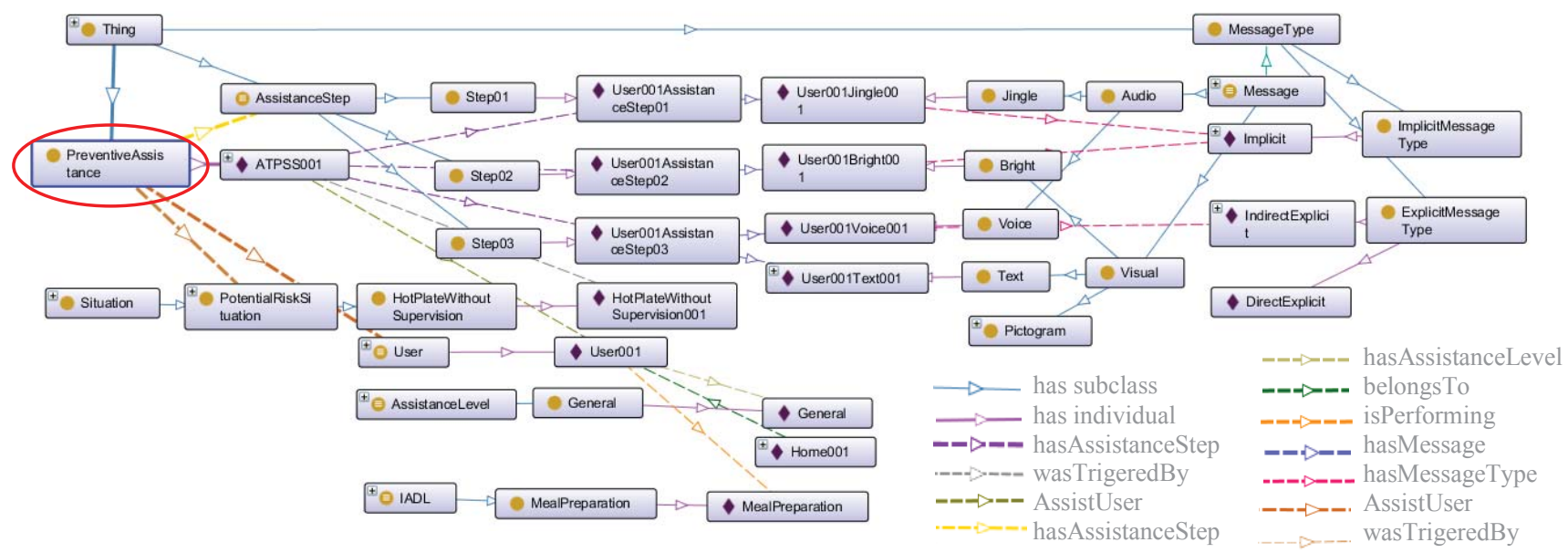

FIGURE 5: PreventiveAssistance class definition in Protégé

\subsection{Providing assistance}

PAS runs along COOK and SS. It uses information in the ontology and reasoning facilities to monitor the user and assist him/her whenever it is necessary. Its life cycle involves 5 main states: Activating, Monitoring, Assisting, Stoppingassistance successful, and Stopping-assistance failed.

Activating. PAS is started when COOK is launched by the user. In this state, PAS retrieves information on the user: preferred interaction mode, specific risk management... Each user has his/her own personalized set of risks to monitor and associated trigger values, e.g. how long he/she can leave the stove unattended. Once activated, it goes to Monitoring state.

Monitoring. In this state, PAS uses information in the ontology (risk contexts specific to the user, current situation, sensor data, user location, etc.) to recognize potential risk contexts during the user's meal preparation. Actually, a potential risk situation is detected when the sensor values resulting from the user's actions in the environment correspond with those defined in a potential risk context description (Table 1). On such detection, it goes to Assisting state.

Assisting. In this state, PAS first generates a preventive assistance tree describing interventions, e.g. tasks PAS should undertake, communication with the user that should take place, e.g. messages that may have to be transmitted to the user, and actions expected from the user. It is described in the form of a preventive assistance tree inspired from Hierarchical Task Networks (HTN) [16]. Preventive assistance is decomposed into tasks named "assistance steps". The current canvas is based on three steps performed sequentially. This allows assistance intervention to progress over a limited period of time along two axes of speech acts: from implicit to explicit content and from indirect to direct speech acts. Each assistance step can contain up to 3 messages that can be transmitted to the user in sequence or in parallel. Message content and type are selected with respect to the user's occupational profile abilities and preferences. Next PAS executes the HTN preventive assistance tree (FIGURE 6). There is a default preventive assistance tree made up of three assistance steps taken in sequence. If the user takes appropriate remedial actions then PAS goes to Stoppingassistance successful state, otherwise to state Stoppingassistance failed.

Stopping-assistance successful. The execution of the preventive assistance tree stops when the user's actions are the ones expected to solve the potential risk situation. PAS takes some action to $\log$ the information and goes to Monitoring state.

Stopping-assistance failed If the preventive assistance tree is fully executed and the user does not resolve the situation in the estimated time, then the autonomous SS intervenes and 
locks the electrical appliance(s) involved in the potential risk situation. PAS intervenes notifying the situation to caregivers. COOK is also locked.

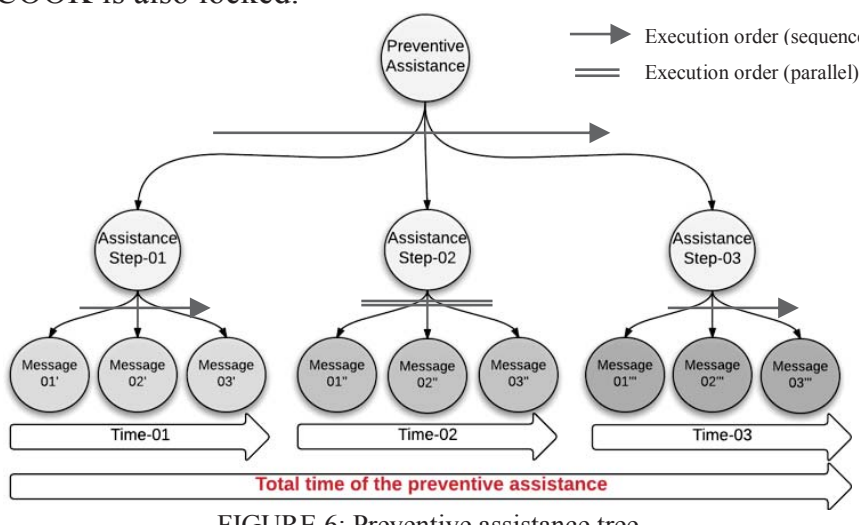

FIGURE 6: Preventive assistance tree

\section{Scenario}

Let's use a scenario to illustrate the functionalities of PAS: potential risk detection, preventive assistance tree instantiation, and execution. In the scenario, a user is cooking something on the stove, then he/she goes in the living room leaving the stove unattended —which is considered as potentially leading to a risk situation if left unattended for 10 minutes. PAS has to make three interventions before the user returns to the kitchen (Steps A to E in Figure 7).

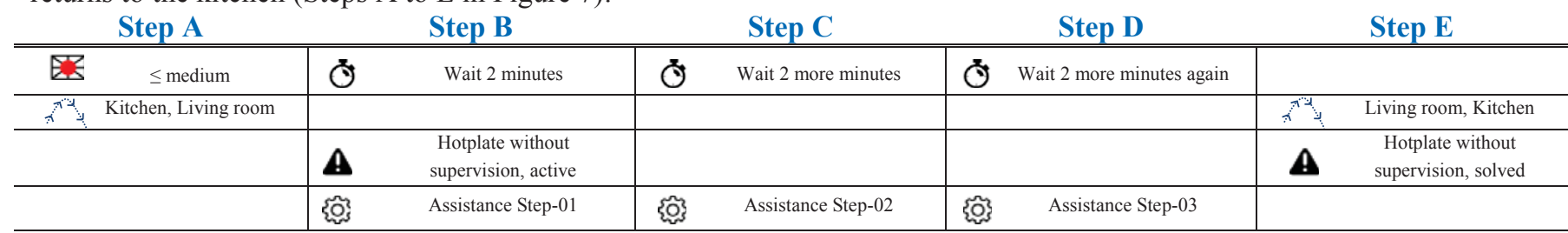

(a) Frédéric Chauvin
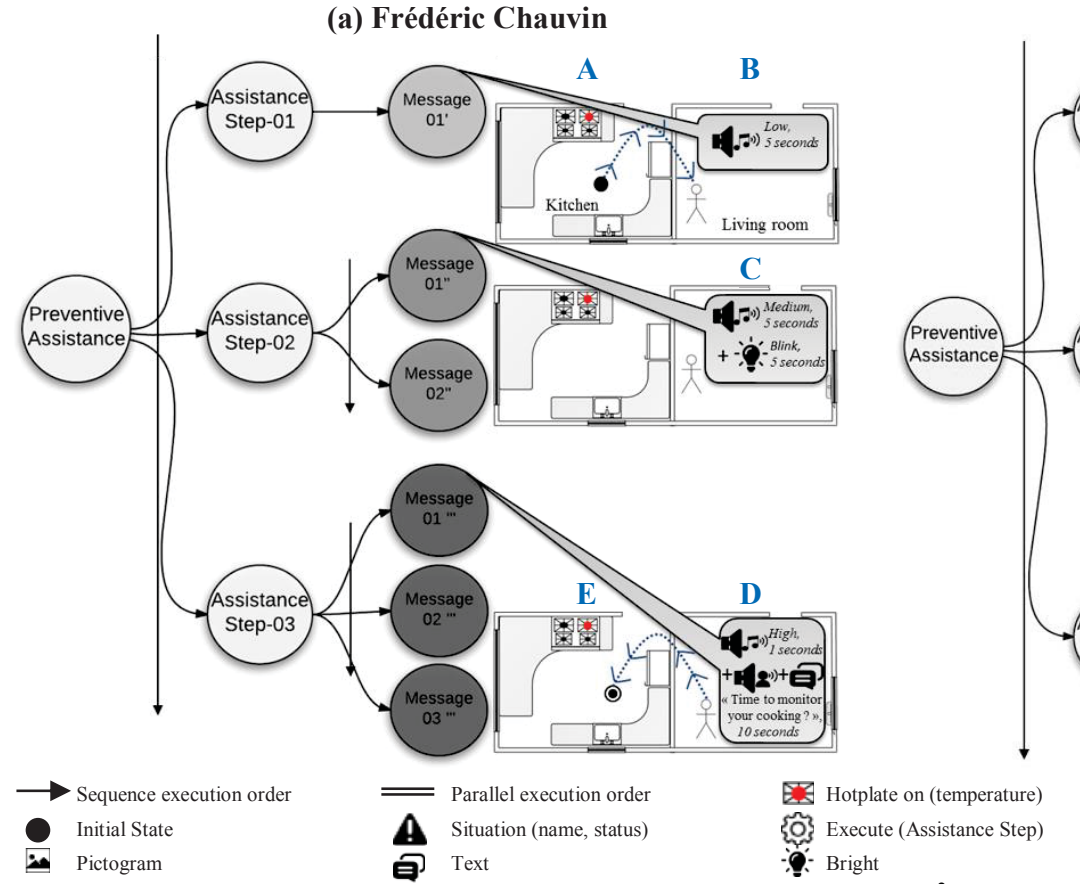

Hotplate on (temperature)

โू్

:- Bright

国 Text

FIGURE 7: Preventive assistance model instantiation ${ }^{3}$ for (a) Frédéric Chauvin (b) Jacques Dupont.
To exemplify variations in assistance, this same scenario will be played involving two different personas. Each persona is a fictitious character exhibiting typical cognitive impairments and behaviors of a severe TBI population [23]. The first persona, Frédéric Chauvin, needs general assistance and guidance from implicit to explicit prompting. The second persona, Jacques Dupont, needs step by step assistance; guidance by explicit prompting. The initial state of the environment will be considered the same for the two personas. Unfolding of the scenario goes as follows (Figure 7):

Step A. Frédéric is cooking; he is using a low- temperature hotplate, and he starts to feel tired. He goes to the living room to take a rest. Thanks to the preventive assistance model, PAS is aware of the state and temperature of the hotplate as well as Frédéric's position inside the home. room. PAS changes the status of the potential risk situation "hotplate without supervision" to "Active" which can conduct to one safety rule SS could trigger after 10 minutes (rule 4 of Table 1). PAS starts an assistance intervention, by instantiating a personalized preventive assistance tree according to the potential risk situation detected and Frédéric's occupational profile (assistance level, preferred communication channel such as audio and/or visual, and his chosen sounds, voice, etc.) (Figure 7a).
Step B. Two minutes later, Frédéric is still in the living 
This preventive assistance tree is then executed using the actuators available at the user's location. The preventive assistance is executed in three sequential assistance steps (Steps B, C, D). If the remedial action is not performed by the persona in the expected period of time, then the next assistance step is executed.

The process is akin for Jacques, but PAS does not generate the same assistance tree since they have different profiles (Figure 7b). Jacques needs detailed assistance since he is more impaired. Let's see the differences in concrete assistance.

For the first intervention (Step B), after two minutes of lying on the sofa, Frédéric is prompted once: a jingle (ImplicitMessageType). This is an implicit message since no specific content is uttered to explain the situation. Jacques is prompted twice: a jingle (ImplicitMessageType) and a pictogram (ImplicitMessageType) emitted in parallel, giving a stronger message that something may go wrong.

For the second intervention, which is provided two minutes after the first one (Step C), Frédéric is prompted twice sequentially: a jingle (ImplicitMessageType) and a blinking light (ImplicitMessageType), the first one aims to attract his attention, the second one aims to indicate the direction to go. The message is more insisting since Frédéric did not react to the first intervention. Similarly, the "message" is also getting more emphatic for Jacques who is prompted by three messages, all played concurrently: a jingle (ImplicitMessageType), a pictogram (ImplicitMessageType), and a text message (indirect ExplicitMessageType).

Frédéric is still not reacting. So for the third intervention occurring two minutes after the second (Step D), Frédéric is prompted three times in sequence: a jingle (ImplicitMessageType), a generic voice message (indirect ExplicitMessageType), and a text message (indirect ExplicitMessageType) Similarly Jacques is also prompted three times in sequence but more firmly: a jingle (ImplicitMessageType), a voice (direct ExplicitMessageType), a text (direct ExplicitMessageType).

Finally, Frédéric (and Jacques) returns to the kitchen and resumes monitoring the hotplate within the expected time (Step E). Thus, the status of the detected situation changes to "Solved" and Frédéric continues to follow instructions from COOK until he completes the meal preparation. If the preventive assistance tree would have been fully executed and Frédéric (or Jacques) would not have performed the expected remedial actions in the expected time, caregivers would have been notified, the autonomous SS would have deactivated the stove, COOK would have been locked, PAS would have changed the status of the risk situation to "Unsolved", notified the detected situation to the user's caregivers, all of them waiting for a caregiver to approve unlocking of the stove and restarting them.

\section{Conclusion}

This paper presented a generic preventive assistance model for individuals with severe TBI. This model is adaptable to any home, IADL, and user occupational profile.
Indeed, we consider that a) a Preventive Assistance System can reduce user exposition to dangerous situations during a meal preparation activity, and, b) a progressive preventive assistance using prompts such as jingles, pictograms, blinking lights, and short voice messages could spark users' awareness about possible risk situations and encourage them to realize remedial actions to solve the detected potential risk situation.

Given the lack of formal models and mechanisms enabling personalized gradual preventive assistance, our work is a first stepping-stone in this direction. Our model is the result of a true and close interdisciplinary collaboration involving various disciplines, caregivers, and users. The use of an ontology was a worthy support for exchanging with experts and clarifying the choices made. These are just first steps. There are still many issues and avenues to explore for further improvements.

The current implementation of COOK includes only static ad-hoc messages and text to prevent safety issues. This implementation can be improved by close interactions with PAS. COOK would gain 1) context-awareness that allows a real-time detection of a potential risk situation, 2) the personalization of message tailoring preventive assistance according to the user's cognitive capacities and preferences (meaningful audio or visual messages) through a configuration tool to set up the preventive assistance parameters based on the model structure, and 3) the generation of a personalized preventive assistance tree based on context-awareness and the user's occupational profile.

Besides, machine learning techniques could also be of benefit to the further development of PAS by bringing improvement and evolution capacities to the user's occupational profile preferences in order to provide to the user more efficient prompting content and strategies.

Finally, the eventual development of a priority management mechanism for potential risk situation occurrences is a must. We must take into account instances when the preventive assistance model is in execution and other potential risk situations are detected.

\section{Acknowledgements}

We gratefully acknowledge the support from the Canadian establishments: Sherbrooke Fire Department, Centre de Réadaptation Estrie (CRE) and its alternative housing unit.

The authors thank Catherine Laliberté, Fanny Le-Morellec and Carolann Fecteau-Mathieu for the management support provided with external and internal entities involved in this research, Pierre-Yves Groussard, Geneviève Lantier, Antoine Vella, Ferhat Abbas and Jeremy Manceaux for their contribution to the problem analysis and early development of the model, and Adrien Barton for the many discussions on ontologies.

This research was funded by the Canadian Institutes of Health Research (CIHR), Natural Sciences and Engineering Research Council of Canada (NSERC), the Research Centre on Aging affiliated to the Université de Sherbrooke, and the French Télécom \& Société numérique Carnot Institute. 


\section{References}

[1] Billette and T. Janz, "Les blessures au Canada: un aperçu des résultats de l'Enquête sur la santé dans les collectivités canadiennes." Stat. Canada, 2011.

[2] C. Dumont, "L'identification des facteurs qui vont favoriser la participation sociale des adultes présentant des séquelles de Traumatisme craniocérébral," 2003.

[3] T. A. Novack, B. A. Bush, J. M. Meythaler, and K. Canupp, "Outcome after traumatic brain injury: Pathway analysis of contributions from premorbid, injury severity, and recovery variables," Arch. Phys. Med. Rehabil., vol. 82, no. 3, pp. 300-305, 2001.

[4] C. Bottari, C. Dassa, C. Rainville, and E. Dutil, "A Generalizability Study of the Instrumental Activities of Daily Living Profile," Arch. Phys. Med. Rehabil., vol. 91, no. 5, pp. 734-742, 2010.

[5] A. R. Rabinowitz and H. S. Levin, "Cognitive Sequelae of Traumatic Brain Injury," Psychiatr. Clin. North Am., vol. 37, no. 1, pp. 1-11, 2014.

[6] A. M. Cook and J. M. Polgar, Cook and Hussey's assistive technologies: principles and practice, Third Edition. 2013.

[7] J. P. Wherton and A. F. Monk, "Technological opportunities for supporting people with dementia who are living at home," Int. J. Hum. Comput. Stud., vol. 66, no. 8, pp. 571-586, Aug. 2008.

[8] N. Bier, C. Bottari, C. Hudon, S. Joubert, G. Paquette, and J. Macoir, "The impact of semantic dementia on everyday actions: evidence from an ecological study.," J. Int. Neuropsychol. Soc., vol. 19, no. 2, pp. 162-72, Feb. 2013

[9] S. Giroux, N. Bier, H. Pigot, B. Bouchard, A. Bouzouane, M. Levasseur, M. Couture, C. Bottari, B. Swaine, P.-Y. Therriault, K. Bouchard, F. Le Morellec, S. Pinard, S. Azzi, M. Olivares, T. Zayani, G. Le Dorze, P. De Loor, A. Thépaut, and B. Le Pévédic, "Cognitive Assistance to Meal Preparation: Design, Implementation, and Assessment in a Living Lab," 2015 AAAI Spring ..., pp. 01-25, 2015.

[10] P. Olivier, G. Xu, A. Monk, and J. Hoey, "Ambient kitchen: designing situated services using a high fidelity prototyping environment," Proc. 2nd Int Conf. Pervasive Technol. Relat. to Assist. Environ., p. 47, 2009.

[11] E. M. Tapia, S. S. Intille, and K. Larson, "Activity recognisation in Home Using Simple state changing sensors," Pervasive Comput., vol. 3001, pp. 158-175, 2004.

[12] A. Coronato and G. Paragliola, "A safe kitchen for cognitive impaired people," Lect. Notes Comput. Sci. (including Subser. Lect. Notes Artif. Intell. Lect. Notes Bioinformatics), vol. 8276 LNCS, pp. 17-25, 2013.

[13] H. Mahajan, "Development and Evaluation of a Smart Cueing Kitchen for Individuals with Cognitive Impairments," Arch. Phys. Med. Rehabil., vol. 94, no. 10, pp. e1-e2, 2013.
[14] K. Tsui and H. Yanco, "Prompting devices: a survey of memory aids for task sequencing," ... Intell. Syst. Better Living, Held ..., 2010.

[15] D. Ann Amin and K. Kannenber, "Occupational Therapy Practice Framework: Domain and Process," Am. J. Occup. Ther., vol. 68, no. Supplement 1, pp. S1-S48, 2014.

[16] S. Russell and P. Norvig, Artificial Intelligence: A Modern Approach, 3rd edition. 2009.

[17] M. Baldauf, S. Dustdar, and F. Rosenberg, "A survey on context-aware systems," Int. J. Ad Hoc Ubiquitous Comput., vol. 2, no. 4, p. 263, 2007.

[18] W3C, “OWL 2 Web Ontology Language." [Online]. Available: https://www.w3.org/TR/owl2-overview/.

[19] Stanford, "Protégé." [Online]. Available: http://protege.stanford.edu/.

[20] L. M. Reeves, J.-C. Martin, M. McTear, T. Raman, K. M. Stanney, H. Su, Q. Y. Wang, J. Lai, J. a. Larson, S. Oviatt, T. S. Balaji, S. Buisine, P. Collings, P. Cohen, and B. Kraal, "Guidelines for multimodal user interface design," Commun. ACM, vol. 47, no. 1, p. 57, 2004.

[21] T. Hart, K. Hawkey, and J. Whyte, "Use of a portable voice organizer to remember therapy goals in traumatic brain injury rehabilitation: a within-subjects trial.," J. Head Trauma Rehabil., vol. 17, no. 6, pp. 556-70, 2002.

[22] J. Searle, Speech Acts an essay in the philosophy of Language. Cambridge University Press, 1969.

[23] M. Olivares, H. Pigot, T. Zayani, N. Bier, C. Bottari, G. Le Dorze, B. Le Pévédic, S. Pinard, B. Swaine, P. Therriault, A. Thépaut, and S. Giroux, "Traumatic Brain Injury Persona: An Interdisciplinary Approach to Designing a Cooking Assistant," Artic. under Rev., 2016. 